\title{
Evidencia in vitro de la utilidad de cloranfenicol y rifampicina para el tratamiento de infecciones sistémicas y meningitis causadas por Streptococcus pneumoniae aislados de niños menores de 5 años en Colombia
}

\author{
Mariluz Hernández, Gloria Isabel Mejía, Hugo Trujillo, Jaime Robledo \\ Facultad de Medicina, Universidad de Antioquia, Medellín, Colombia. \\ 2 Unidad de Bacteriología y Micobacterias, Corporación para Investigaciones Biológicas, Medellín, Colombia.
}

Actualmente, Streptococcus pneumoniae resistente a penicilina es un patógeno común en infecciones pediátricas, lo cual plantea dificultades en el tratamiento. La rifampicina y el cloranfenicol se han recomendado como antibióticos útiles y como alternativas en estos casos, pues son menos costosos y más accesibles a las comunidades de recursos limitados. Su uso, sin embargo, puede estar limitado por los niveles de resistencia encontrados en diferentes poblaciones. El objetivo del presente estudio fue determinar la concentración inhibitoria mínima (CIM) y la concentración bactericida mínima (CBM) a la rifampicina y al cloranfenicol en aislamientos de S. pneumoniae pertenecientes a un grupo recolectado entre 1994 a 1996 de niños menores de 5 años con infección sistémica y meningitis. Se estudiaron 107 aislamientos, 60 de ellos resistentes a penicilina y 47 sensibles, 53 de los cuales fueron aislamientos de LCR. La CIM y la CBM se realizaron de acuerdo con las recomendaciones de la NCCLS; se utilizó S. pneumoniae ATCC 49619 como control. Se consideraron como sensibles al cloranfenicol los aislamientos con $\mathrm{CIM}=4 \mu \mathrm{g} / \mathrm{ml}$ y resistentes, con $\mathrm{CIM}=8 \mu \mathrm{g} / \mathrm{ml}$; sensibles a rifampicina con $\mathrm{CIM}=1 \mu \mathrm{g} / \mathrm{ml}$ y resistentes con $\mathrm{CIM}=4 \mu \mathrm{g} / \mathrm{ml}$. La CBM se determinó con la menor concentración del antibiótico que inhibió el crecimiento del 99,9\% del inóculo. Se encontró resistencia al cloranfenicol en $20,5 \%$ de los 107 aislamientos estudiados. En el grupo resistente a la penicilina, $28 \%$ fue resistente al cloranfenicol y $11 \%$ en el grupo sensible a la penicilina. La CBM para el cloranfenicol fue $>4 \mu \mathrm{g} / \mathrm{ml}$ en $28 \%$ de los aislamientos sensibles a la penicilina y en $60 \%$ de los resistentes. No se encontraron aislamientos resistentes a la rifampicina; sin embargo, 2 aislamientos mostraron CBM $>1 \mu \mathrm{g} / \mathrm{ml}$ y una CBM=16 $\mu \mathrm{g} / \mathrm{ml}$. Los aislamientos de LCR presentaron la CIM y la CBM más altas que el grupo total de aislamientos. Los datos sugieren que en Colombia el cloranfenicol no se debe usar como terapia empírica para infecciones invasivas por neumococo en niños menores de 5 años. La rifampicina combinada con otros antibióticos en el tratamiento de estas infecciones puede ser una alternativa. Es necesario realizar otros estudios para conocer el significado que tendrían los bajos niveles de CBM a la rifampcina encontrados en algunos de los aislamientos, para la eficiacia de estos tratamientos.

Palabras claves: Streptococcus pneumoniae, resistencia a penicilina, cloranfenicol, rifampicina

Effectiveness of the antibiotics chloramfenicol and rifampin in the treatment of Streptococcus pneumoniae-induced meningitis and systemic infections

Streptococcus pneumoniae, a common pathogen in pediatric infections, has become resistant to penicillin and make these infections difficult to treat. Rifampin and chloramphenicol have been recommended as alternative therapies, since they are less costly and more accessible to communities with limited resources. However, their use may be restricted by the differing levels of resistance found in target populations. The objective was to determine minimal inhibitory concentration (MIC) and minimal bactericidal concentration (MBC) for chloramphenicol and rifampin in strains of $S$. pneumoniae. These strains were newly isolated from children under age 5 that had demonstrated systemic infections and meningitis. A subgroup of 107 isolates of 
S. pneumoniae was selected from 324 strains isolated during a period of 2 years (1994-1996). Among these isolates, 60 were penicillin-resistant and 47 were susceptible; 53 isolates were from children with meningitis. MIC and MBC for chloramphenicol and rifampicin were obtained by standard methods recommended by the National Committee for Clinical Laboratory Standards (NCCLS). S. pneumoniae ATCC strain 49619 served as the control. An isolate was considered susceptible to chloramphenicol when $\mathrm{MIC}=4 \mu \mathrm{g} / \mathrm{ml}$ and resistant when $\mathrm{MIC}=8 \mu \mathrm{g} / \mathrm{ml}$. A strain was considered susceptible to rifampin when $\mathrm{MIC}=1 \mu \mathrm{g} / \mathrm{ml}$ and resistant when $\mathrm{MIC}=4 \mu \mathrm{g} / \mathrm{ml}$. MBC was determined by recording the lower concentration of the antibiotic that inhibited $99.9 \%$ of the initial inoculum. Chloramphenicol resistance was found in $21 \%$ of the 107 isolates. In the group susceptible to penicillin, $11 \%$ were resistant to chloramphenicol and in the group resistant to penicillin $28 \%$ was resistant to chloramphenicol as well. MBC was found $>4 \mu \mathrm{g} / \mathrm{ml}$ in $28 \%$ of the isolates susceptible to penicillin and in $60 \%$ of the resistant isolates. No isolates were found resistant to rifampin. However, 2 penicillin resistant isolates showed $C B M>1 \mu \mathrm{g} / \mathrm{ml}$ to rifampin, and one with $C I M=1 \mu \mathrm{g} / \mathrm{ml}$ had a MBC to rifampicin of $16 \mu \mathrm{g} / \mathrm{ml}$. Meningitis isolates showed higher CIM and CBM than the group of total isolates. These data suggest that chloramphenicol is not recommended for invasive infections caused by $S$. pneumoniae in Colombia. Rifampin is a more effective therapy in combination with other antibiotics for treatment of this kind of infections. Further studies are necessary to clarify the significance of low levels of MBC to rifampin found in some strains, since this may affect the efficacy of therapies that include this antibiotic.

Key words: Streptococcus pneumoniae, penicillin resistance, rifampin, chloranphenicol

La prevalencia actual de Streptococcus pneumoniae resistente a la penicilina ha convertido las infecciones comunes en pediatría, como las otitis y las meningitis bacterianas agudas, en entidades de difícil manejo. El tratamiento de estas infecciones es ahora un verdadero reto para el clínico, ya que el neumococo puede ser resistente no sólo a la penicilina, sino también a las cefalosporinas de tercera generación como la cefotaxima y la ceftriaxona, consideradas por muchos años los medicamentos de elección (1).

Los programas de vigilancia coordinados por la Organización Panamericana de la Salud en el Sistema Regional de Vacunas (SIREVA/OPS) y los programas nacionales de vigilancia de meningitis bacteriana aguda y de infección respiratoria han documentado la prevalencia de neumococo resistente a la penicilina en Colombia. Entre 1994 y 2000 , la sensibilidad disminuida a la penicilina en S. penumoniae aislado en niños menores de 5 años se estimó en $26,1 \%$, del cual $11,8 \%$

\footnotetext{
Correspondencia:

Jaime Robledo, Unidad de Bacteriología y Micobacterias, Corporación para Investigaciones Biológicas, Carrera $72^{\mathrm{a}}$ No. 78B-141, Medellín, Colombia jrobledo@cib.org.co
}

Recibido: 21/01/03; aceptado: 18/11/03 correspondió a resistencia intermedia y $14,3 \%$ a resistencia de alto nivel. En estos aislamientos, la resistencia al cloranfenicol fue de $11,1 \%$ y a la ceftriaxona de $13,6 \%$ (2).

En las meningitis causadas por neumococo resistente a la penicilina y a las cefalosporinas de tercera generación, se han recomendado tratamientos combinados de vancomicina con cefalosporinas de tercera generación (ceftriaxona o cefotaxima) o de rifampicina con cefalosporinas de tercera generación o con vancomicina $(3,4)$.

En muchas áreas de países en desarrollo, las cefalosporinas de tercera generación y la vancomicina son costosas y no están fácilmente disponibles; por tanto, su utilización en el tratamiento inicial de las meningitis es limitada. Otros antibióticos menos costosos y más accesibles que pudieran ser una alternativa en estos casos, son la rifampicina y el cloranfenicol. Particularmente, este último ha sido de amplia utilización en el tratamiento inicial de las meningitis, no obstante su toxicidad medular y la descripción de aislamientos resistentes (5). Actualmente y debido a lo anterior, sólo se recomienda su utilización en forma segura cuando se han demostrado concentraciones bactericidas mínimas (CBM) menores o iguales a $4 \mu \mathrm{g} / \mathrm{ml}$ (5). La rifampicina se ha recomendado en conjunto 
con la vancomicina en el tratamiento de las meningitis causadas por neumococo, cuando se documentan niveles altos de resistencia a cefalosporinas de tercera generación (6). En los modelos experimentales de meningitis, la rifampicina se ha demostrado con actividad bactericida y tiene un efecto aditivo con la vancomicina (7).

El objetivo del presente estudio fue determinar las concentraciones inhibitoria mínima y bactericida mínima al cloranfenicol y a la rifampicina, de islamientos de $S$. pneumoniae causantes de infección sistémica y de meningitis en niños menores de 5 años, con el propósito de conocer la utilidad potencial de estos antibióticos en el tratamiento de la meningitis bacteriana aguda en nuestro medio.

\section{Materiales y métodos}

Se estudiaron 107 aislamientos de S. pneumoniae, escogidos al azar dentro del grupo de 324 obtenidos durante el estudio de vigilancia de resistencia a penicilina de $S$. pneumoniae en Colombia, los que fueron suministrados por el Grupo de Microbiología del Instituto Nacional de Salud. De éstos, se estudiaron 60 de los 61 resistentes a penicilina $(\mathrm{CIM} \geq 0,12 \mu \mathrm{g} / \mathrm{ml})$ y 47 sensibles $(C I M \leq 0,06 \mathrm{mg} / \mathrm{ml})$, que fueron escogidos al azar dentro del grupo de sensibles de este mismo estudio (2). En el grupo total de 107 aislamientos, estaban incluidos 53 aislamientos de líquido cefalorraquídeo, 25 en el grupo de sensibles a penicilina y 28 en el grupo de resistentes.

Se determinó la concentración inhibitoria mínima al cloranfenicol y a la rifampicina por microdilución en caldo de acuerdo con estándares del NCCLS; se utilizó la cepa ATCC 49619 de S. pneumoniae para los controles respectivos (8). Se consideró que un aislamiento era sensible al cloranfenicol cuando la CIM era $\leq 4 \mu \mathrm{g} / \mathrm{ml}$ y resistente $\geq 8 \mu \mathrm{g} / \mathrm{ml}$ y sensibles a rifampicina cuando la CIM era $\leq 1$ $\mu \mathrm{g} / \mathrm{ml}$ y resistente si $\geq 4 \mu \mathrm{g} / \mathrm{ml}$ (9).

La CBM se realizó mediante subcultivos de la CIM y 4 diluciones adicionales por encima de ésta; su valor fue dado de acuerdo con la determinación de la menor concentración de los antibióticos que era capaz de eliminar $99,9 \%$ de las bacterias en el inóculo inicial (10).

\section{Resultados}

Se demostró resistencia al cloranfenicol en 20,5\% de los 107 aislamientos estudiados. En el grupo de aislamientos sensibles a la penicilina, $11 \%$ fue resistente al cloranfenicol, mientras que en el grupo de los aislamientos resistentes a la penicilina, la resistencia al cloranfenicol fue de $28 \%(p=0,02)$. En el grupo de aislamientos de meningitis, la resistencia al cloranfenicol fue de $22 \%$ y $28 \%$ para los sensibles y resistentes a penicilina, respectivamente.

No se observaron diferencias en $\mathrm{la}_{\mathrm{CIM}}$ al cloranfenicol en los aislamientos sensibles a penicilina, entre el grupo total y el de los aislamientos de meningitis. Sin embargo, se observaron $\mathrm{CIM}_{90}$ mayores en el grupo de aislamientos de meningitis tanto en el grupo de sensibles como en los resistentes a penicilina. (cuadro 1).

La CBM ${ }_{50}$ y la $\mathrm{CBM}_{90}$ de cloranfenicol fue similar en el grupo total y el grupo de meningitis, tanto para los aislamientos sensibles a penicilina $=4$ $\mu \mathrm{g} / \mathrm{ml}$ y $16 \mu \mathrm{g} / \mathrm{ml}$, como para los resistentes a penicilina $=8 \mu \mathrm{g} / \mathrm{ml}$ y $=32 \mu \mathrm{g} / \mathrm{ml}$, respectivamente (cuadro 1). La concentración bactericida mínima al cloranfenicol fue $>4 \mu \mathrm{g} / \mathrm{ml}$ en $28 \%$ de los aislamientos sensibles y en $60 \%$ de los resistentes a penicilina; en estos últimos, $15 \%$ presentó una CBM más de 4 veces la CIM para este antibiótico.

Cuadro 1. Sensibilidad al cloranfenicol en neumococos invasivos y causantes de meningitis aislados en menores de 5 años en Colombia (1994-1996), de acuerdo con la sensibilidad a la penicilina.

\begin{tabular}{|c|c|c|c|c|}
\hline \multirow[t]{3}{*}{ Cloranfenicol } & \multicolumn{2}{|c|}{$\begin{array}{c}\text { Aislamientos } \\
\text { sensibles a } \\
\text { penicilina }\end{array}$} & \multicolumn{2}{|c|}{$\begin{array}{c}\text { Aislamientos } \\
\text { resistentes a } \\
\text { penicilina }\end{array}$} \\
\hline & $\begin{array}{c}\text { LCR } \\
(n=25)\end{array}$ & $\begin{array}{c}\text { Total } \\
(\mathrm{n}=47)\end{array}$ & $\begin{array}{c}\text { LCR } \\
(n=28)\end{array}$ & $\begin{array}{c}\text { Total } \\
(n=60)\end{array}$ \\
\hline & \multicolumn{4}{|c|}{$\mu \mathrm{g} / \mathrm{ml}$} \\
\hline $\mathrm{CIM}_{50}$ & 2 & 2 & 4 & 4 \\
\hline $\mathrm{CIM}_{90}^{50}$ & 8 & 4 & 32 & 16 \\
\hline $\mathrm{CBM}_{50}^{90}$ & 4 & 4 & 8 & 8 \\
\hline $\mathrm{CBM}_{90}$ & 16 & 16 & 32 & 32 \\
\hline
\end{tabular}

CIM: concentración inhibitoria mínima

CBM: concentración bactericida mínima 
No se encontró resistencia a la rifampicina en ninguno de los aislamientos estudiados. $\mathrm{La} \mathrm{CIM}_{50}$ y la $\mathrm{CIM}_{90}$ para este antibiótico fueron bajas en los aislamientos sensibles y en los resistentes a la penicilina, tanto en el grupo total como en el de aislamientos de meningitis.

Sin embargo, se encontró una tendencia a tener CIM mayores en el grupo de aislamientos meníngeos resistentes a la penicilina. En la $\mathrm{CBM}_{90}$ no se encontraron diferencias entre el grupo total de aislamientos y los de meningitis, excepto en el grupo de meningitis para los aislamientos resistentes a penicilina $(=0,5 \mu \mathrm{g} / \mathrm{ml}$ en el grupo total vs $1,0 \mu \mathrm{g} / \mathrm{ml}$ en el grupo de meningitis) $y$ sólo en este grupo se observaron $\mathrm{CBM}_{90}$ mayores de 1,0 $\mu \mathrm{g} / \mathrm{ml}$. Tres de los 4 aislamientos resistentes a la penicilina en los que se demostró una CBM a rifampicina mayor de 4 veces la CIM, fueron meníngeos (cuadro 2).

\section{Discusión}

La resistencia a la penicilina en $S$. pneumoniae es importante en Colombia y está en aumento, tal como lo muestran los datos de la vigilancia de la resistencia del neumococo a los antibióticos, realizada por los programas de vigilancia coordinados por la OPS en el Sistema Regional de Vacunas (SIREVA/OPS) y por el Laboratorio Nacional de Referencia del Instituto Nacional de Salud. Para 1994, esta resistencia era de $10 \%$ y para el 2000 habia llegado a $38,2 \%$ (2). La importancia de esta tendencia implica la necesidad de considerar antibióticos alternativos para tratar las infecciones invasivas causadas por este microorganismo.

Cuadro 2. Sensibilidad a la rifampicina en neumococos invasivos y causantes de meningitis aislados en menores de 5 años en Colombia (1994-1996), de acuerdo con la sensibilidad a la penicilina.

\begin{tabular}{|c|c|c|c|c|}
\hline \multirow[t]{3}{*}{ Rifampicina } & \multicolumn{2}{|c|}{$\begin{array}{c}\text { Aislamientos } \\
\text { sensibles a } \\
\text { penicilina }\end{array}$} & \multicolumn{2}{|c|}{$\begin{array}{l}\text { Aislamientos } \\
\text { resistentes a } \\
\text { penicilina }\end{array}$} \\
\hline & $\begin{array}{c}\text { LCR } \\
(n=25)\end{array}$ & $\begin{array}{c}\text { Total } \\
(n=47)\end{array}$ & $\begin{array}{c}\text { LCR } \\
(n=28)\end{array}$ & $\begin{array}{c}\text { Total } \\
(n=60)\end{array}$ \\
\hline & \multicolumn{4}{|c|}{$\mu \mathrm{g} / \mathrm{ml}$} \\
\hline $\mathrm{CIM}_{50}$ & 0,12 & 0,12 & 0,12 & 0,12 \\
\hline $\mathrm{CIM}_{90}$ & 0,25 & 0,25 & 0,5 & 0,25 \\
\hline $\mathrm{CBM}_{90}$ & 0,5 & 0,5 & 1,0 & 0,5 \\
\hline
\end{tabular}

Es importante señalar que los aislamientos de neumococo en Colombia, además de ser resistentes a penicilina, pueden también serlo a otros antibióticos como ceftriaxona (13,6\%), trimetoprim/sulfa $(40,1 \%)$ y eritromicina $(4,6 \%)$ (2). La resistencia al cloranfenicol encontrada en el presente estudio de $20,5 \%$ es mayor a la presentada por datos más recientes, $11 \%$ (2), lo cual puede estar influenciado por la selección de los aislamientos probados en el estudio, diferencias en el tamaño de la muestra o por diferencias en el período de tiempo abarcado. Estos datos, en conjunto con los del presente estudio, no respaldan el uso del cloranfenicol como una terapia empírica adecuada para el tratamiento de las infecciones invasivas y graves por neumococo en menores de 5 años en Colombia, puesto que el $28 \%$ de los aislamientos resistentes a la penicilina lo fueron también al cloranfenicol. Esto se hace más evidente en el grupo de aislamientos de meningitis, ya que en éstos se observó una resistencia mayor a este antibiótico aún siendo sensibles a penicilina.

Los datos de CBM al cloranfenicol encontrados enfatizan la recomendación de no utilizar este antibiótico como alternativa de tratamiento en meningitis puesto que el $49 \%$ de los aislamientos meníngeos presentó una $\mathrm{CBM}>4 \mu \mathrm{g} / \mathrm{ml}$, por encima de lo recomendado para garantizar un tratamiento efectivo (5).

La rifampicina ha sido recomendada para el tratamiento de meningitis en combinación con cefalosporinas de tercera generación o vancomicina (6). Este antibiótico penetra bien al sistema nervioso central por ser una droga lipofílica y, en niños, sus niveles son en promedio $1 \pm 0,5$ con un rango entre $0,3-1,9 \mu \mathrm{g} / \mathrm{ml}(12,13)$. La ausencia de resistencia del neumococo a la rifampicina demostrada en este estudio, respalda el uso de este antibiótico concomitantemente con la vancomicina, en la terapia asociada de infecciones invasivas por neumococo resistente a la penicilina, especialmente en meningitis.

No obstante, el presente estudio mostró 2 aislamientos resistentes a la penicilina del grupo de meningitis, con CBM a la rifampicina $>1 \mu \mathrm{g} /$ $\mathrm{ml}$. Estos niveles están muy cerca de los alcanzados por el antibiótico en líquido 
cefalorraquídeo y no permiten una terapia segura. El hallazgo de 3 aislamientos meníngeos resistentes a la penicilina con CBM a rifampicina 4 veces mayor que la CIM, sugiere la existencia de tolerancia en estos aislamientos, lo cual confirma la precaución que se debe tener en estos casos para utilizar este antibiótico en el tratamiento de las meningitis por $S$. pneumoniae. Sin embargo, se desconoce cual prodría ser la respuesta clínica con la terapia combinada vancomicina-rifampicina o cefalosporinasrifampicina, cuando la CBM esté en estos niveles. Por consiguiente, el empleo de rifampicina en estos casos debe darse bajo protocolos de investigación controlados hasta que la evidencia permita definir que la respuesta clínica es adecuada.

Es importante considerar que la resistencia del neumococo a la rifampicina puede ser más frecuente en poblaciones particulares, como son los pacientes infectados con el virus de la inmunodeficiencia humana y aquéllos bajo terapia antituberculosa $(14,15)$. Son necesarios estudios más amplios y dirigidos a estas poblaciones para confirmar la importancia de esta resistencia en el país.

En resumen, los datos encontrados en el presente estudio no apoyan el uso de cloranfenicol como terapia alternativa cuando se sospeche una meningitis por neumococo resistente a penicilina en niños colombianos menores de 5 años. Los datos sugieren que el uso de rifampicina asociada con otros antibióticos puede ser una terapia alternativa en esta entidad, basado en la ausencia de resistencia de los aislamientos estudiados. No obstante, la presencia de aislamientos con tolerancia a la acción bactericida del antibiótico pueden comprometer el éxito de la terapia.

\section{Agradecimientos}

Esta investigación fue cofinanciada por la Universidad de Antioquia y la Corporación para Investigaciones Biológicas, Medellín.

El protocolo nacional para serotipificación de $S$. pneumoniae fue apoyado por la Organización Panamericana de la Salud a través de su programa especial para vacunas e inmunización (SVI) y por el Sistema Regional de Vacunas (SIREVA) con apoyo de la Canadian International Development Agency (CIDA).

Al Grupo de Microbiología del Instituto Nacional de Salud y al grupo de estudio del neumococo en Colombia.

\section{Referencias}

1. Feigin RD, Pearlman E. Bacterial meningitis beyond the neonatal period. En: Feigin-Cherry, editor. Textbook of pediatric infectious diseases. Fourth edition. Philadelphia: WB Saunders Company; 1998. p.400-29.

2. Agudelo $\mathrm{Cl}$, Sanabria $\mathrm{OM}$, Ovalle OM, Castañeda E, Grupo Colombiano de trabajo en Streptococcus pneumoniae. Vigilancia por el laboratorio de Streptococcus pneumoniae, aislado de procesos invasores en niños menores de 5 años: actualización de los datos 1994-2000. Inf Quinc Epidemiol Nac 2001; 7:97-104.

3. Friedland I, McCracken GH. Management of infections caused by antibiotic resistant Streptococcus pneumoniae. N Engl J Med 1994;331:377-82.

4. París MM, Ramilo O, McCracken GH. Management of meningitis caused by penicillin-resistant Streptococcus pneumoniae. Antimicrob Agents Chemother 1995; 39:2171-5.

5. Friedland IR, Klugman KP. Failure of chloramphenicol therapy in penicillin resistant pneumococcal meningitis. Lancet 1992;339:405-8.

6. Kaplan SL. Management of pneumococcal meningitis. Pediatric Infect Dis J 2002;21:589-91.

7. Martínez-Lacasa J, Cabellos C, Martos A, Fernández A, Tubau F, Viladrich PF, Linares J, Gudiol F. Experimental study of the efficacy of vancomycin, rifampin, and dexomethasone in the therapy of pneumococcal meningitis. J Antimicrob Chemother 2002; 49:507-13.

8. National Committee for Clinical Laboratory Standards. Methods for dilution antimicrobial susceptibility tests for bacteria that grow aerobically. Fourth edition; approved standard. Wayne, Pennsilvania: NCCLS; 1997.

9. National Committee for Clinical Laboratory Standards. Performance standards for antimicrobial susceptibility testing. Twelfth Informational Supplement. NCCLS document M100-S12. Wayne, Pennsylvania: NCCLS; 2002.

10. Knapp C, Moody JA. Tests to assess bactericidal activity. En: Isenberg H, editor. Clinical microbiology procedures handbook. Washington, D.C.: American Society for Microbiology; 1992. p.5.16.1-5.16.33.

11. Agudelo Cl, Castañeda E, Gallego CR, Jaramillo E, Ramírez E, Lozada C, et al. Vigilancia por el laboratorio de Streptococcus pneumoniae, aislado de procesos 
invasores en niños menores de 5 años, 1994-1998. Inf Quinc Epidemiol Nac 1998;3:250-5.

12. Scheld WM. Drug delivery to the central nervous system: general principles and relevance to therapy for infections of the central nervous system. Rev Infect Dis $1989 ; 11(\mathrm{~S} 7): \mathrm{S} 166-1690$.

13. Klugman K, Fiedland I, Bradley J. Bactericidal activity against cephalosporin-resistant Streptococcus pneumoniae in cerebrospinal fluid of children with acute bacterial meningitis. Antimicrob Agents Chemother 1995;39:1988-92.

14. García-Arenzana J, Montes M, Pérez-Trallero E. Are rifampin-resistant Streptococcus pneumoniae strains a consequence of the increase in cases of tuberculosis? Clinic Infect Dis 1994;19:360-1.

15. Klugman K. Pneumococcal resistance to antibiotics. Clin Microb Rev 1990;3:171-96. 